\title{
Turbulence in Core-Collapse Supernovae
}

\author{
David Radice ${ }^{1,2}$, Ernazar Abdikamalov ${ }^{3}$, Christian D. Ott ${ }^{4}$, \\ Philipp Mösta跴, Sean M. Couch ${ }^{6,7,8}$, and \\ Luke F. Roberts ${ }^{6,8}$. \\ ${ }^{1}$ School of Natural Sciences, Institute for Advanced Study, 1 Einstein Drive, \\ Princeton, NJ 08540, USA \\ 2 Department of Astrophysical Sciences, 4 Ivy Lane, Princeton University, \\ Princeton, NJ 08544, USA \\ ${ }^{3}$ Department of Physics, School of Science and Technology, Nazarbayev \\ University, Astana 010000, Kazakhstan \\ 4 TAPIR, Walter Burke Institute for Theoretical Physics, Mailcode 350-17, \\ California Institute of Technology, Pasadena, CA 91125, USA \\ ${ }^{5}$ Department of Astronomy, 501 Campbell Hall \#3411, University of California \\ at Berkeley, Berkeley, California 94720, USA \\ ${ }^{6}$ Department of Physics and Astronomy, Michigan State University, East \\ Lansing, MI 48824, USA \\ 7 Department of Computational Mathematics, Science, and Engineering, \\ Michigan State University, East Lansing, MI 48824, USA \\ 8 National Superconducting Cyclotron Laboratory, Michigan State University, \\ East Lansing, MI 48824, USA
}

\begin{abstract}
Multidimensional simulations show that non-radial, turbulent, fluid motion is a fundamental component of the core-collapse supernova (CCSN) explosion mechanism. Neutrino-driven convection, the standing accretion shock instability, and relic-perturbations from advanced stages of nuclear burning can all impact the outcome of core collapse in a qualitative and quantitative way. Here, we review the current understanding of these phenomena and their role in the explosion of massive stars. We also discuss the role of protoneutron star convection and of magnetic fields in the context of the delayed neutrino mechanism.
\end{abstract}

\section{Introduction}

The formation of an iron core at the center of evolved massive stars, i.e., with zero-age main-sequence masses of $\sim 10 M_{\odot}$ or more, is a robust prediction of stellar evolution theory. It is also well established that, once it reaches a critical mass of $\sim 1.4 M_{\odot}$, the iron core becomes dynamically unstable and succumbs to its self-gravity. The collapse proceeds until the center reaches nuclear density $\simeq 2.7 \times 10^{14} \mathrm{~g} \mathrm{~cm}^{-3}$. Then, the equation of state (EOS) stiffens because of the repulsive component of the strong nuclear force. The inner part of the iron core, that is in sonic contact during the collapse, halts its contraction and bounces back. A protoneutron star (PNS) is thus formed. The outer part of the iron core, that is infalling supersonically, rams into it producing a strong shock wave.

In the case of the most common variety of core-collapse supernovae (CCSNe), this shock wave is expected to expand toward the stellar surface, depositing energy

$\ddagger$ NASA Einstein Fellow 
and unbinding material, ultimately powering the explosion. However, this process is far from straightforward. Within $\sim 20 \mathrm{~ms}$ of its formation, the shock wave loses about two Bethes $\left(1 \mathrm{~B} \equiv 10^{51} \mathrm{erg}\right)$ of energy due to the neutrino shock-breakout burst. Subsequently, as the shock moves out in mass, it loses energy at the rate of $8.8 \mathrm{MeV}$ per nucleon $\left(\sim 1.7 \mathrm{~B}\right.$ per $\left.0.1 M_{\odot}\right)$ as it dissociates iron-group nuclei into free nucleons. Eventually, within a few tens of milliseconds, the shock stalls and turns into a standing accretion shock stagnating at a radius between 100 and 200 kilometers. For the explosion to be successful, some physical mechanism must revive the shock within a timescale of $\sim 1$ second. Otherwise, the PNS would collapse to form a black hole. Understanding this mechanism is one of the most pressing problems in astrophysics.

The leading theoretical model for shock revival is the so-called delayed neutrino mechanism [1, 2]. According to this mechanism, it is the absorption of neutrinos radiated from the PNS and its cooling mantle that revives the shock. All modern simulations show the formation of a layer behind the shock, the so-called gain region, with net neutrino heating. Whether this is the mechanism solely responsible for the explosion is still uncertain. For instance, we now know that for most progenitors the delayed neutrino mechanism does not work in spherical symmetry $3[8]$. However, successful explosions have been obtained in multiple dimensions thanks to the presence of hydrodynamical instabilities, such as the standing accretion shock instability (SASI) [9, 10] and neutrino-driven convection [11,14]. These instabilities break the spherical symmetry of the problem and generate turbulence. These two effects, asphericities of the flow and turbulence, are now recognized as being crucial for successful explosions. Indeed, as shown in Fig. 1, turbulence is present over most of the region interior to the shock.

The kinematic viscosity in the region immediately below the shock is dominated by neutron scattering and is very small [15, implying an extremely high Reynoldsnumber flow $\left(\operatorname{Re} \approx 10^{17}\right.$; $\left.[15]\right)$. An important ramification for radiationhydrodynamics CCSN simulations is that they need to be 3D. They also need to have sufficiently high resolution to capture the turbulent flow according to the implicit large eddy simulation (ILES) paradigm; whereby the bulk of the large-scale energycontaining eddies are well resolved and numerical dissipation is used as an effective turbulent model at small scales [16 18. In 1D (spherical symmetry) there is only radial flow. In 2D (axisymmetry), turbulence exhibits an inverse cascade that transfers kinetic energy to large scales [19] where it can artificially help the explosion [20 22].

The purpose of this article is to review our current understanding of the role of turbulence and other fluid instabilities in the explosion mechanism of CCSNe. We refer to the recent reviews by [29 32 for more broad overviews of CCSN theory. The rest of this article is organized as follows. In Section 2, we focus on the development of neutrino-driven convection and its role in the explosion mechanism. Section 3 is dedicated to the SASI. In Section 4 we consider the evolution of perturbations, originating in convective nuclear burning shells, as they are advected and amplified during core collapse. We discuss in more detail the role that pre-SN perturbations can have in aiding the explosion in Section 5. Section 6 is dedicated to the role of PNS convection. We review the possible role of magnetohydrodynamic (MHD) effects for the explosion of regular CCSNe in Section 7. Finally, we conclude in Section 8 with a brief summary and a discussion of future prospectives in the study of turbulence in CCSNe. 


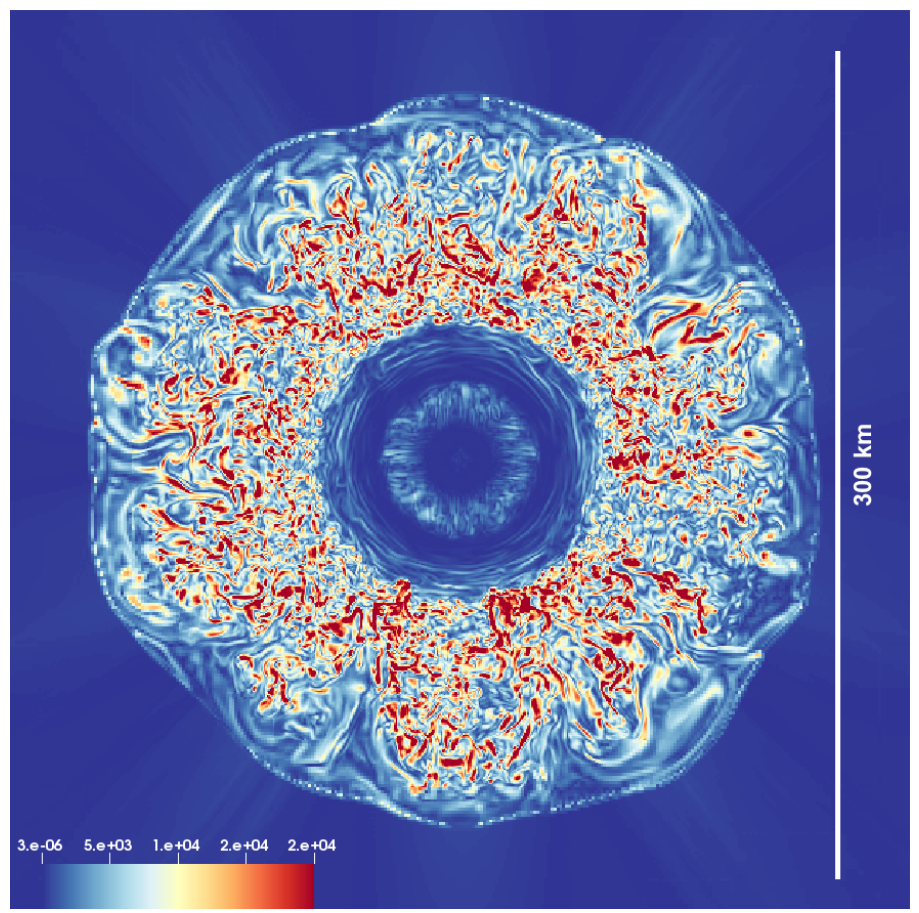

Figure 1. Regions of intense vorticity during the stalled-shock phase of the explosion of a CCSN. The color code shows the amplitude of the vorticity. The data are taken at 210 milliseconds after bounce for a $20-M_{\odot}$ progenitor. The progenitor model was computed with MESA 23] 24], while the simulation was performed in 3D using the FLASH code with M1 neutrino transport 2527. Turbulent convection immediately behind the shock is clearly visible and is characterized by the presence of small tubular structures with intense vorticity. Similar structures are known to characterize incompressible turbulence in periodic domains 28]. The vortical structures near the core are due to PNS convection.

\section{Neutrino-Driven Convection}

\subsection{The Development of Convection}

Because of the geometric dilution of the neutrino radiation field, the net heating rate per baryon has a maximum within the gain layer and decreases outwards. This naturally leads to the formation of an unstable (negative) entropy gradient. The gain region is unstable to convection according to both the Schwarzschild [33] and the Ledoux 34 criteria.

According to the Ledoux criterion, in the case of a static background, the growth rate of small convective eddies is given by the imaginary part of the Brunt-Väisälä frequency:

$$
\Omega_{\mathrm{BV}}^{2}=\frac{g}{\rho}\left(\frac{\partial \rho}{\partial p}\right)_{s, Y_{e}}\left[\left(\frac{\partial p}{\partial s}\right)_{\rho, Y_{e}} \frac{\partial s}{\partial r}+\left(\frac{\partial p}{\partial Y_{e}}\right)_{\rho, s} \frac{\partial Y_{e}}{\partial r}\right],
$$

where the electron fraction $Y_{e}$ is used to express the composition of the flow, $s$ is its entropy per baryon, and $g$ is the gravitational acceleration. 

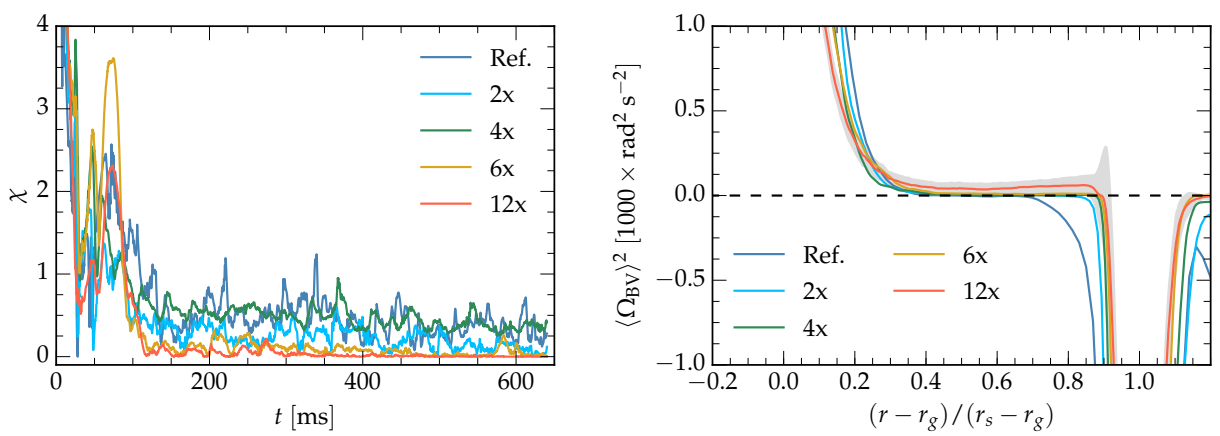

Figure 2. Left panel: evolution of Foglizzo's $\chi$, Eq. 22. Right panel: time and angle averaged Brunt-Väisälä frequency $\Omega_{\mathrm{BV}}$ as a function of a normalized radial coordinate. The results are from the $3 \mathrm{D}$ high-resolution, semi-global, parameterized, neutrino-driven convection simulations presented in 18. Here, $\chi$ is computed from the angle-averaged $\Omega_{\mathrm{BV}}$ and velocity, $r_{g}$ is the radius at which the angle-averaged net neutrino heating rate first becomes positive, and $r_{s}$ is the shock radius. The initial value of $\chi$ is 5.33 , but it drops significantly once convection is fully developed and $\left\langle\Omega_{\mathrm{BV}}\right\rangle^{2}$ is positive over most of the gain region. The final value of $\chi$ also decreases with resolution.

Classically, as soon as $\Omega_{\mathrm{BV}}^{2}<0$ convection is expected to develop. However, as pointed out by Foglizzo et al. [14, in the case of the gain layer in CCSNe, there is an additional condition that needs to be satisfied for convection to develop: the growth rate of the convective instability must be large enough to ensure that buoyant plumes can grow sufficiently before being advected through the gain region. This condition is expressed in terms of the non-dimensional parameter

$$
\chi=\int \frac{\left|\Omega_{\mathrm{BV}}\right|}{\left|v_{r}\right|} \mathrm{d} r
$$

where the integral is restricted to those regions of the gain layer where $\Omega_{\mathrm{BV}}^{2}$ is negative. $\chi$ can be interpreted as the number of e-folding times experienced by a buoyant perturbation before being accreted into the cooling layer above the PNS. Many numerical simulations have shown that, when $\chi \gtrsim 3$, convection develops 14, 15, 22, 35-42. On the other hand, the interpretation of $\chi$ in the presence of large deviations of the background flow from spherical symmetry is less clear. First, since Eq. (2) is non-linear, computing $\chi$ using angle and time averaged flow variables gives results that can be significantly different from its direct evaluation as a volume integral [39. This is because, in this case, the value of $\chi$ can be dominated by points with small radial velocities. Differences are also likely to arise depending on whether $\chi$ is computed using the average Brunt-Väisälä frequency, e.g., 39, or the Brunt-Väisälä frequency of the averaged flow, e.g., 40. Second, numerical simulations suggest that in the presence of sufficiently large perturbations convection might develop also for small values of $\chi$ 35, 43. Finally, once convection is fully developed, $\chi$ often decreases to below the critical value of three 22,39 .

For example, Fig. 2 shows that if $\chi$ is computed from the angle averaged $\Omega_{\mathrm{BV}}$ and radial velocity, then the development of convection tends to drive $\chi$ toward zero. In particular, the left panel Fig. 2 shows the evolution of $\chi$ from a series of parametrized semi-global neutrino driven convection simulations [18. The reference resolution is $\Delta r \simeq 3.8 \mathrm{~km}$ and $\Delta \phi=\Delta \theta=1.8^{\circ}$. The other simulations have their resolution 
increased (grid spacing decreased) by factors 2, 4, 6, and 12. The initial value of $\chi$ is 5.33 , but, as soon as convection is fully developed, $\chi$ drops significantly and it reaches essentially zero in the highest resolution runs. Despite the vanishing value of $\chi$, convection is fully developed for the entire duration of the simulations. However, the flow re-arranges so that $\left\langle\Omega_{\mathrm{BV}}\right\rangle^{2} \gtrsim 0$ almost everywhere in the gain layer (right panel of Fig. 2). The only exceptions are the points where the angle averaging crosses the shock surface and $\left\langle\Omega_{\mathrm{BV}}\right\rangle$ is not particularly meaningful (these points are not considered when computing $\chi$ from Eq. 2]).

Clearly, the interpretation of Foglizzo's $\chi$ once the flow has broken the spherical symmetry is difficult and its value is not necessarily indicative of whether convection or SASI is dominating. On the other hand, the value of $\chi$ shortly after bounce is predictive of the onset of convection. Foglizzo's criterion also shows that convection should become dominant for models with low accretion rates and/or high neutrino luminosities. This has been extensively confirmed by many $3 \mathrm{D}$ simulations, e.g., 15, 20, 22, 36, 40, 43, 50.

\subsection{The Impact of Convection}

The importance of convection in the gain layer below the shock has been recognized for many years, see e.g., [51. The results from some of the earliest multi-dimensional (2D) simulations suggested that convection might be strong enough to trap energy into the gain region [11, 52. According to these simulations, the temperature of the gas below the shock would then continuously increase due to neutrino absorption until a critical threshold after which the explosion would set in. This was the so-called convective engine model. However, the results from more advanced simulations, e.g., starting from [12, 13, 53, questioned these earlier studies and showed that the impact of convection is important, but more subtle.

Modern simulations show that once convection develops, a new equilibrium is typically reached with no net accumulation of mass or energy in the gain region. Instead, the explosion, if successful, is likely to develop in a more nuanced way if the right conditions, often expressed in terms of neutrino energy deposition and accretion rate 54, are met. In other words, neutrino-driven convection is not able to completely trap material in the gain region and create a run-away accumulation of energy. However, it can aid the explosion by creating more favorable conditions for the shock to run away, for instance, when the accretion rate suddenly drops as compositional interfaces cross the shock.

One way convection aids the explosion is by entraining some of the gas, which, as a consequence, has more time to absorb neutrinos [12, 44, 55, 56]. Equivalently, convection decreases the typical advection timescale

$$
t_{\mathrm{adv}}=\frac{M_{\text {gain }}}{\dot{M}},
$$

bringing it closer to the timescale needed to heat the material in the gain region sufficiently to unbind it [57/59].

Another possibility is that if convection is sufficiently strong, it could drive a redistribution of entropy leading to conditions that are more favorable for explosion [51]. This effect was quantified by Yamasaki \& Yamada [60 in terms of its reduction on the critical neutrino luminosity [54], the neutrino luminosity beyond which the shock is able to overcome the ram pressure of the accreting material. 

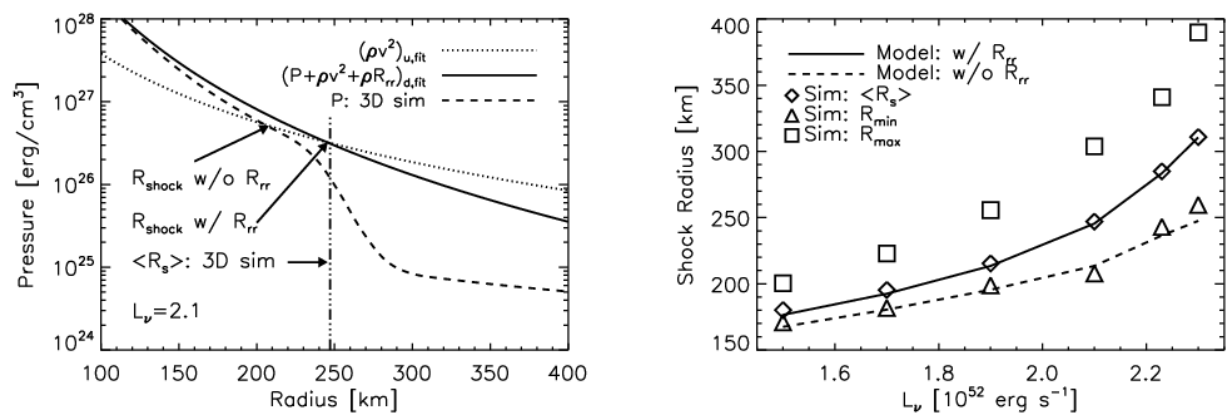

Figure 3. Left panel: power-law fits of the upstream and downstream components of Eq. (5). The location of the average shock radius $\left\langle R_{s}\right\rangle$ and of the predictions from Eq. (4), which does not account for the turbulent pressure, and from Eq. 5, which includes the effect of turbulent pressure, are also indicated. Right panel: minimum, maximum, and average shock radii in parametrized 3D simulations of neutrino-driven convection with different neutrino luminosities. Also shown are the predictions from Eqs. (4) and (5). Reprinted from J. Murphy, J. Dolence, and A. Burrows, The Dominance of Neutrino-Driven Convection in Core-Collapse Supernovae, The Astrophysical Journal 771, 52 (2013) [45, (C) AAS. Reproduced with permission.

They showed that if convection is able to eliminate the radial gradient of the angleaveraged specific entropy, then the critical luminosity is effectively decreases by a factor of two. On the other hand, simulations have shown that, in conditions typical of CCSNe, neutrino-driven convection is not sufficiently vigorous to realize the scenario hypothesized by Yamasaki \& Yamada [18, 37, 43, 44, 61, 62, and the reduction in the critical neutrino luminosity is smaller than what they predicted.

Neutrino-driven convection behind the shock is turbulent and the chaotic motion of the fluid can result in an effective additional pressure support behind the stalled shock. This was first pointed out by Murphy et al. [45] and later confirmed and extended by Couch \& Ott [21] and by Radice et al. [18. Following the original argument by Murphy et al. [45], we consider the Rankine-Hugoniot momentum condition for a standing accretion shock,

$$
\rho_{d} v_{d}^{2}+p_{d}=\rho_{u} v_{u}^{2}+p_{u},
$$

where $\rho$ is the density, $v$ the radial velocity, and $p$ the pressure. We have also denoted with $\cdot_{d}$ and $\cdot_{u}$ the downstream and upstream quantities respectively.

In the presence of turbulence, [45] suggested to modify equation (4) in a way akin to a Reynolds decomposition and write it as

$$
\rho_{d} v_{d}^{2}+\rho_{d} R_{r r}+p_{d}=\rho_{u} v_{u}^{2}+p_{u},
$$

where $R_{r r}$ is the radial component of the Reynolds stress tensor,

$$
R_{i j}=\left\langle\delta v_{i} \delta v_{j}\right\rangle,
$$

and $\delta v_{i}$ is the difference between the fluid velocity and the angle-averaged radial velocity of the flow. Although not entirely rigorous, Eq. (5) has been shown to be well verified in numerical simulations if angular averages are used to compute the respective quantities [18, 21, 45, 63; see Fig. 3. In that Figure, reproduced from [45], the shock location in 3D parametrized neutrino-driven convection simulations is 
compared with the prediction from Eq. (5). It is found that the effective pressure support generated by the turbulence accounts for an increase in the shock radius of $\sim 25 \%$ [45]. Furthermore, Couch \& Ott [21] showed that the effective pressure support due to turbulence can be as large as $\sim 50 \%$ of the thermal pressure.

Couch \& Ott 21] also showed that the transition to explosion in CCSNe is associated with an increase in the total turbulent energy in the gain region, defined as

$$
E_{\text {turb }}=\frac{1}{2} \rho|\delta \mathbf{v}|^{2} .
$$

The importance of turbulent energy can be better understood by recasting Eq. (5), following 64 ]

$$
\rho_{d} v_{d}^{2}+\left(\gamma_{\mathrm{th}}-1\right) \rho_{d} \epsilon_{\mathrm{th}}+\left(\gamma_{\mathrm{turb}}-1\right) \rho_{d} \epsilon_{\mathrm{turb}}=\rho_{u} v_{u}^{2}+p_{u},
$$

where $\epsilon_{\text {th }}$ is the specific internal energy downstream of the shock, $\gamma_{\text {th }} \simeq 4 / 3$ is the adiabatic index for a radiation pressure dominated gas, and $\epsilon_{\text {turb }}=E_{\text {turb }} / \rho_{d}$ is the specific turbulent energy density. $\gamma_{\text {turb }}$ is an effective adiabatic index which is equal to $5 / 3$ for isotropic turbulence and to 2 for anisotropic turbulence with $R_{r r} \simeq R_{\theta \theta}+R_{\phi \phi}$. The latter case is commonly realized in convection simulations, e.g., [18, 21, 45, 65], and should be the relevant case for CCSNe. Equation (8) illustrates that turbulent kinetic energy contributes to the overall pressure support behind the shock. More importantly, since $\gamma_{\text {turb }}>\gamma_{\text {th }}$, Eq. (8) shows that turbulent kinetic energy is a more "valuable" form of energy than thermal energy, since it can more effectively overcome the ram pressure ahead of the shock. The ratio $\epsilon_{\mathrm{turb}} / \epsilon_{\mathrm{th}}$ is then a crucial quantity for the revival of the shock.

Another formulation of Eq. (8) was proposed by Müller \& Janka [63. Instead of the turbulent energy, they computed the turbulent pressure from the rms Mach number of the turbulence

$$
\left\langle\mathcal{M}^{2}\right\rangle=\frac{2 E_{\text {kin }, \theta}}{M_{\text {gain }} c_{s, d}^{2}} \simeq \frac{\epsilon_{\text {turb }}}{c_{s, d}^{2}},
$$

$c_{s, d}$ being the sound speed downstream of the shock. With this definition the total pressure support behind the shock (turbulent plus thermal) is given by

$$
p=p_{\text {th }}\left(1+\gamma_{\text {th }}\left\langle\mathcal{M}^{2}\right\rangle\right) \simeq p_{\text {th }}\left(1+\frac{4}{3}\left\langle\mathcal{M}^{2}\right\rangle\right)
$$

Radice et al. [18 presented a more formal derivation of Eq. (5), which they reformulated as a global integral condition implicitly determining the location of the shock. Their analysis confirmed the previous interpretation of the importance of the effective turbulent pressure. Furthermore, they found that the non-radial fluid motion due to turbulence also contributes significantly to the effective pressure support of the flow. This is because, even though the angular momentum of the flow is conserved, the fluid still experiences a net centrifugal force.

More recently, Mabanta \& Murphy [66] considered the impact of turbulence on the explosion conditions using a new semi-analytic model. They extended the work of Yamasaki \& Yamada 60] by combining the neutrino-driven convection model developed by Murphy \& Meakin 62 with the steady-state model of Murphy \& Dolence [67. They pointed out that neutrino-driven convection is able to tap into the gravitational potential energy associated with the unstable stratification of the material behind the shock. According to their model, buoyancy would transform this 
potential energy into kinetic energy, which would then be transformed into thermal energy by turbulent dissipation. Mabanta \& Murphy [66] argued that the subsequent enhanced thermalization of the flow would be the most important consequence of turbulence for the explosion mechanism of CCSNe. However, this effect has not yet been investigated using multi-dimensional simulations, so its quantitative impact remains unclear.

Clearly, all of the effects of neutrino-driven convection just discussed are deeply connected. The increased residence time of fluid elements in the gain region, the convective luminosity, the turbulent dissipation, and the effective turbulent pressure support, can all be described using the Reynolds decomposition, e.g., [18, 62, of the mass, energy, and momentum conservation equations respectively.

\subsection{Turbulent Energy Cascade}

On the basis of the previous discussion, we can conclude that the ratio between turbulent and thermal pressure is an important parameter affecting the dynamics of the shock revival in neutrino-driven CCSNe. This ratio in turn is set by the efficiency with which kinetic energy is transferred to small scales by the turbulence cascade, where it is dissipated into heat. Understanding how the turbulent cascade operates in neutrino-driven convection and how well it is captured in finite-resolution simulations is important to be able to quantify the role of turbulence in the explosion and the fidelity of the numerical models.

The turbulent energy spectrum is the main diagnostic used to study the turbulent cascade [15, 21, 22, 37, 44, 46, 68, 69. Most investigators have focused on the transverse turbulent velocity spectrum

$$
E(\ell)=\sum_{m=-\ell}^{\ell}\left|\int \sqrt{\rho} v_{t} Y_{\ell}^{m} \mathrm{~d} \Omega\right|^{2},
$$

where $Y_{\ell}^{m}$ are the spherical harmonics, $v_{t}$ is the non-radial part of the velocity, and the integral is evaluated on a shell at a fixed radius. Another quantity of interest is the specific turbulent energy spectrum

$$
E(k)=\frac{1}{2} \sum_{i=1}^{3} \int_{\mathbb{R}^{3}} \delta(|\mathbf{k}|-k) \widehat{\delta v_{i}^{*}}(\mathbf{k}) \widehat{\delta v_{i}}(\mathbf{k}) \mathrm{d} \mathbf{k},
$$

where $\widehat{r}$ is the Fourier transform, defined as

$$
\widehat{\delta v_{i}}(\mathbf{k})=\int_{\mathbb{R}^{3}} \delta v_{i}(\mathbf{x}) e^{2 \pi \mathbf{i} \mathbf{k} \cdot \mathbf{x}} \mathrm{d} \mathbf{x}
$$

and.$^{*}$ denotes the complex conjugation. Finally, $\delta v_{i}$ is defined as in Eq. 66. Apart from the normalization factor, this quantity coincides with the velocity powerspectrum, which is also the Fourier transform of the two-point correlation function of the velocity [70].

At scales that are well separated from those at which turbulence is driven by neutrinos and dissipated by microphysical processes, the statistical properties of turbulence in steady state, including the spectra, are expected to be universal [70. At these intermediate scales, in the so-called inertial range, the spectra $E(\ell)$ and $E(k)$ should become self-similar power laws [70. For example, in Kolmogorov's classical theory of turbulence $E(k) \propto k^{\alpha}$ with index $\alpha=-5 / 3$. Note that, in the limit of large 
$\ell$, and since $\rho \simeq$ const at a fixed radii, $E(k) \propto k^{\alpha}$ is equivalent to $E(\ell) \propto \ell^{\alpha}$, e.g., [71, chapter 21.

In the phenomenology associated with Kolmogorov's theory the energy flux across scales is constant in the inertial range. Consequently, the energy dissipation rate in well developed turbulence only depends on the large scale dynamics, which provides the "inflow" boundary condition for the energy transport to small scale. Consequently, the presence of a well resolved inertial range is regarded as a sufficient condition to ensure that a numerical simulation is capturing the turbulent dissipation of energy from the largest eddies.

One possible issue is that Kolmogorov's theory of turbulence may not necessarily apply to neutrino-driven convection. Indeed, the flow in CCSNe is affected by nuclear reactions and neutrino irradiation, effects that are not considered in the classical theory. Moreover, turbulence in the postshock region of CCSNe is anisotropic, as gravity breaks the local rotational symmetry of the fluid equations [18, 21, 45, 62, 69, mildly compressible (reaching pre-explosion Mach numbers of $\simeq 0.3-0.5$ [72, 73]), and driven at multiple scales by buoyancy.

Nevertheless, the plasma flow in the gain region is well described using classical hydrodynamics. This is because the plasma constituents have characteristic crosssections of $\sim 10^{-24} \mathrm{~cm}^{2}$. Given the typical temperatures $\sim 1 \mathrm{MeV}$ and densities $\sim 10^{8} \mathrm{~g} \mathrm{~cm}^{-3}$ in the gain region, this implies mean free paths around $10^{-7} \mathrm{~cm}$ and thermodynamical equilibration timescales of few femtoseconds. These scales are many orders of magnitude smaller than those of interest for the modeling of the explosion, so the conditions for the validity of the hydrodynamical description are fully realized. Moreover, momentum exchange due to neutrinos in the gain region is unlikely to damp turbulence due to the long neutrino mean free paths compared to the typical size of eddies in the inertial range [15].

Furthermore, anisotropic and mildly compressible flows have been shown to follow Kolmogorov's theory in other contexts, e.g. 64, 74, 75. The effect of buoyancy has been considered by Goldreich \& Keeley [76] who argued that Kolmogorov's phenomenology should still apply, because turbulent stresses become dominant over buoyancy forces at sufficiently small scales. Here, we present a revised and generalized version of their argument.

Consider eddies with typical size $l$ (not to be confused with the spherical harmonic index $\ell$ ), s.t. $\eta \ll l \ll L$, where $L$ is the energy injection scale, i.e., the size of the gain region, and $\eta$ is the dissipation scale. The buoyancy forces acting on these eddies scale as the entropy fluctuations at the scale of the eddies:

$$
F_{l}^{B} \sim \delta s_{l} .
$$

Inertial forces scale as $\S$

$$
F_{l}^{I} \sim \frac{\left(\delta v_{l}\right)^{2}}{l} .
$$

Let us assume that velocity and entropy fluctuations scale as power laws of

$$
\left(\delta v_{l}\right)^{2} \sim l^{2 \beta}, \quad \delta s_{l} \sim l^{\gamma} .
$$

Note that from Eq. 12 and the properties of the Fourier transform, it follows that the power-spectrum of the velocity is also a power law with index $\alpha=-2 \beta-1$, e.g.,

$\S$ Note that these are a manifestation of the $(\mathbf{v} \cdot \nabla) \mathbf{v}$ term in the Euler's equations.

$\|$ Equivalently, we assume the existence of an inertial range. 


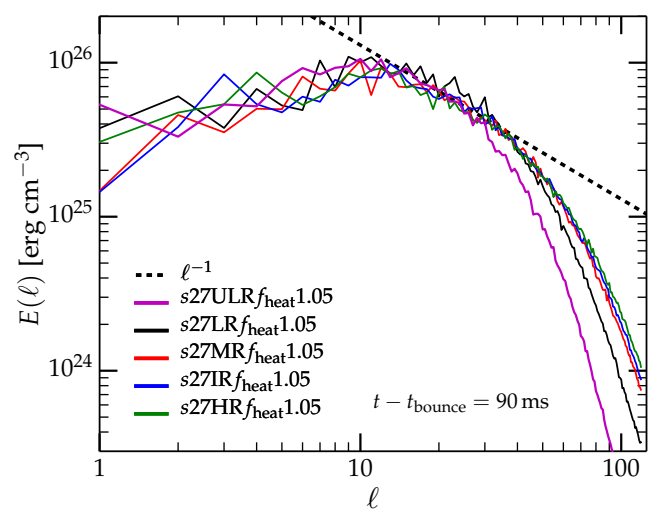

Figure 4. Transverse energy spectra at different resolutions for one of the models from Abdikamalov et al. 15. Note that the spectra have a rather shallow $\ell^{-1}$ dependency at intermediate scales of $10 \lesssim \ell \lesssim 40$. Reprinted from E. Abdikamalov, C. D. Ott, D. Radice, L. F. Roberts, R. Haas, C. Reisswig, P. Mösta, H. Klion, and E. Schnetter, Neutrino-Driven Turbulent Convection and Standing Accretion Shock Instability in Three-Dimensional Core-Collapse Supernovae, The Astrophysical Journal 808, 70 (2015) 15], (C) AAS. Reproduced with permission.

[70. Under these assumptions, the ratio between inertial and buoyant forces acting on eddies with typical scale $l$ is

$$
\frac{F_{l}^{I}}{F_{l}^{B}} \sim l^{2 \beta-\gamma-1},
$$

which shows that inertial forces dominate on sufficiently small scales as long as

$$
2 \beta<\gamma+1 \text {. }
$$

This condition is verified for turbulence with Kolmogorov's spectrum, where $\beta=\gamma=$ $1 / 3$ suggesting that a flow satisfying Eq. (18) will develop a Kolmogorov spectrum at scales $\eta \ll l \ll l^{\star}, l^{\star}$ being the scale at which $F_{l^{\star}}^{I} \sim F_{l^{\star}}^{B}$ over one eddy turnover timescale $\tau_{l}=l / \delta v_{l}$.

We stress that Eq. (18) is satisfied for most values of $\beta$ and $\gamma$ in the physically relevant range $0<\beta, \gamma<1$ (note that $\beta \geq 1$ or $\gamma \geq 1$ would imply smooth, laminar velocity or entropy fields, respectively, e.g., [70]). On the basis of this argument, we can conclude that buoyancy should become negligible at small scales $l \ll l^{\star}$ and deviations from Kolmogorov scaling should not be expected, unless compressibility or other non-ideal effects become important, or Eq. 18 is violated.

Hanke et al. 37. claimed to observe a turbulent energy spectrum with $\alpha \simeq-5 / 3$. Similar results where later reported by Handy et al. 69 and Summa et al. 777. However, later simulations employing Cartesian grids and higher resolutions found shallower power-spectra with $\alpha \simeq-1$ [15, 21, 22, 44]. See Fig. 4 for an example from Abdikamalov et al. [15. Several authors (e.g., [22, 44]) have argued that the $\alpha=-1$ scaling observed in 3D simulations could be due to the physical nature of the postshock turbulent flow that deviates significantly from the assumptions of Kolmogorov turbulence. However, this is unlikely on the basis of the argument we presented above. Furthermore, a scaling with $\alpha=-1$ is unphysical because it would 


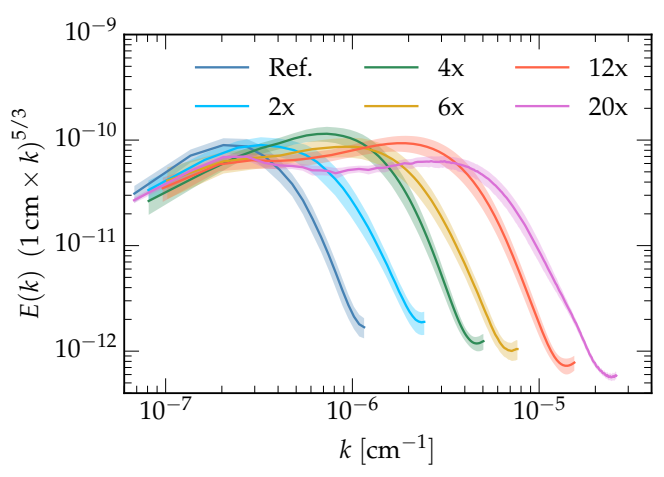

Figure 5. Compensated velocity power-spectra at increasing resolutions in $3 \mathrm{D}$, semi-global, parameterized, neutrino-driven convection simulations. As the resolution is increased, the turbulent specific kinetic energy spectrum approaches the $k^{-5 / 3}$ slope predicted by Kolmogorov's theory. Reprinted from D. Radice, C. D. Ott, E. Abdikamalov, S. M. Couch, R. Haas, and E. Schnetter, NeutrinoDriven Convection in Core-Collapse Supernovae: High-Resolution Simulations, The Astrophysical Journal 820, 76 (2016) [18. (C) AAS. Reproduced with permission.

predict infinite turbulent kinetic energy in the limit of infinite resolution and zero viscosity.

Abdikamalov et al. 15 argued that a common issue in these studies is that the resolution requirements for the recovery of an inertial range had been severely underestimated. Their interpretation is corroborated by the results of local simulations in periodic cubic domains, which showed that resolutions between $512^{3}$ and $1024^{3}$ are needed to recover an inertial range 64, 74, 78. In contrast, typical global 3D CCSN simulations have resolutions roughly equivalent to those of a $64^{3}$ local simulation [15].

In the light of local simulation results, Abdikamalov et al. [15] suggested that the $\ell^{-1}$ spectra found by [15, 21, 22, 44] are the result of the so-called bottleneck effect 79 83. This is a phenomenon arising because of the indirect influence of the (numerical) viscosity on the dynamics at large scale, which results in a build up of energy at wavenumbers close to the dissipation scale. The presence of a numerical bottleneck effect is also likely to result in an artificially large ratio $\epsilon_{\mathrm{turb}} / \epsilon_{\mathrm{th}}$, which would create more favorable conditions for the explosion, as tentatively confirmed by Abdikamalov et al. 15.

In this context, the $\ell^{-5 / 3}$ spectra from Hanke et al. 37. and Handy et al 69. might be interpreted as arising from a misidentification of the dissipation range and inertial ranges. Indeed, the spectra from Hanke et al. [37] appear to become shallower with increasing resolution. This would be expected if the putative inertial range with logarithmic slope $-5 / 3$ was actually a part of the exponentially decaying dissipation range.

More recently, Radice et al. [18] performed a very extensive resolution study of a parametrized neutrino-driven convection model. Their results are reproduced in Fig. 5. They found shallow velocity power spectra at their coarsest resolution, which was intermediate between the medium and the high resolution of Hanke et al. [37. As they increased the resolution they eventually recovered Kolmogorov scaling, but only at a resolution $\simeq 16$ times higher than the highest resolution of Hanke et al. 37. These 


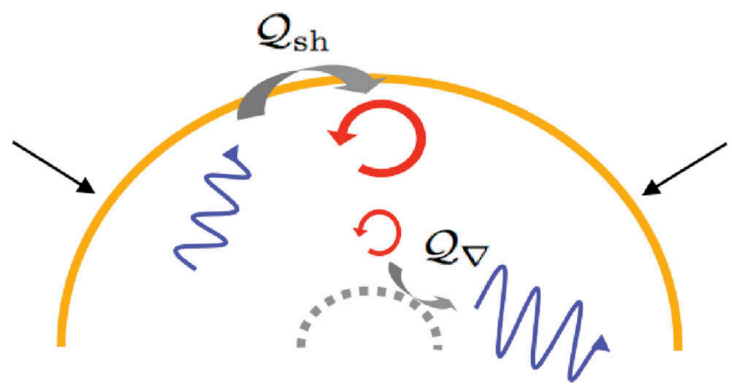

Figure 6. Schematic represenation of the advective-acoustic instability underlying SASI. An entropy/vorticity perturbation is created at the shock front (orange half-circle) by the accretion flow. This perturbation is advected purely radially (red) to the edge of the protoneutron star (indicated by a dashed gray half-circle). There, it is strongly decelerated and converted into an upward pressure perturbation (i.e., acoustic wave) that has radial and transverse components (blue). Reprinted from J. Guilet \& T. Foglizzo, On the linear growth mechanism driving the standing accretion shock instability, Monthly Notices of the Royal Astronomical Society 421, 546 (2012) 92, (C) J. Guilet \& T. Foglizzo. Reproduced with permission.

results provide additional support to the interpretation by Abdikamalov et al. [15] of the $k^{-1}$ spectra seen in many simulations as being due to the insufficient resolution, although a definite confirmation would require the development of techniques for the measurement of the kinetic energy flux accross scales. See, e.g., 64 for a study of this kind in the context of local simulations. Radice et al. [18, also provided solid numerical evidence for Kolmogorov's phenomenology in neutrino-driven convection. However, the idealized simulations of Radice et al. [18] did not include the feedback of convection on the neutrino luminosity, which might give raise to a new phenomenology. This limitation needs to be addressed by future studies.

\section{SASI-Generated Turbulence}

The standing accretion shock instability (SASI) was discovered by Blondin et al. 9 , who performed simplified 2D hydrodynamic simulations of stalled ("standing") accretion shocks. They found that the stalled shock develops an oscillatory instability dominated by an (in terms of spherical harmonics) $\ell=1$ "sloshing" mode. Later studies in 3D showed the prevalence of an azimuthal $m=1$ spiral mode (e.g., [15, 22, 38, 41, 84 86]), which is enhanced by rotation (e.g., [87 89]).

In a series of papers [10, 14, 35, 60, 90,92, Foglizzo and colleagues identified SASI as an advective-acoustic instability (though see 93, 94 for a purely acoustic interpretation). In the advective-acoustic scenario depicted in Fig. 3. a small entropy or vorticity perturbation enters through the shock and is advected with the subsonic accretion flow toward the PNS. At the steep density gradient marking the PNS edge, it is decelerated, causing an outward propagating pressure (i.e., acoustic) wave with radial and transverse components.

In the non-linear regime of SASI, its oscillations reach large amplitudes and cause large spatially and temporally varying expansions and recessions of the shock front. The postshock entropy depends on the preshock velocity in the frame of the shock. The smaller the shock radius, the higher the preshock velocity and the higher the 
postshock entropy. Hence, SASI-driven variations in the shock radius lead to radial entropy gradients from which turbulent convection grows [35, 68, 95, 96. Because of the lateral and azimuthal SASI shock motions, the radial preshock accretion flow impacts the shock at an oblique angle, leading to deflection and large nonradial velocity components. In the highly non-linear regime, non-radial streams from different phases of a SASI cycle collide, forming shear-regions, secondary shocks, and supersonic accretion funnels [35, 95, 97. All these non-linear SASI features create conditions susceptible to turbulence and simulations show that fully turbulent (i.e., as turbulent as permitted by the employed resolution) postshock flow can develop from SASI even in CCSN cores that are initially stable to neutrino-driven convection (e.g., [35, 41, 68, 98).

In $2 \mathrm{D} / 3 \mathrm{D}$ hydrodynamic simulations, SASI is observed to saturate in strength/amplitude when the aforementioned non-linear flow features appear. Guilet et al. [96] analyzed the saturation behavior of SASI and proposed that saturation sets in when a parasitic instability is able to grow fast enough to compete with SASI by impeding the advective-acoustic cycle. They found that both the Rayleigh-Taylor instability (growing on entropy gradients) and the Kelvin-Helmholtz instability (growing in shear flows) can act as such a parasitic instability, but that the Rayleigh-Taylor instability may be the dominant saturation agent. In any case, both parasitic instabilities are well known to result in turbulent flow. This suggests that turbulence is a natural consequence of SASI, which is confirmed by simulations (e.g., [15, 22, 35, 68).

Extensive research went into understanding the interplay of SASI and neutrinodriven convection, often with a focus on the question of which of the two instabilities is dominant in CCSNe. Because of the advective nature of the postshock CCSN flow, neutrino-driven convection can grow only if seed perturbations are large enough, the entropy gradient is steep enough, and/or the advection timescale is long enough (cf. Section 2.1, Eq. 2, and 14, 35). SASI growth, on the other hand, is fastest if the advection timescale is short [14, 35. CCSN simulations with conditions in which neutrino-driven convection grows fast and is strong and sustained do not appear to develop large-amplitude SASI (e.g., [15, 22, 43, 45]). In other simulations, e.g., those in which neutrino heating is weak or that use a progenitor with a particularly high accretion rate (e.g., [15, 22, 38]), neutrino-driven convection may fail to develop or may be present only temporarily. In these scenarios, SASI eventually dominates.

While turbulence appears to be a natural consequence of both instabilities, it remains to be clarified if SASI-induced turbulence is different in character from neutrino-driven turbulent convection. If it is related predominantly to entropy gradients and the Rayleigh-Taylor instability [35, 96, then its properties may be expected to be similar to turbulence resulting from neutrino-driven convection. This is supported by the turbulence analyses of recent detailed 3D CCSN simulations with SASI (e.g., [15, 22]). On the other hand, Endeve et al. 68, found in their 3D simulations that SASI-induced turbulence is driven predominantly by shear flows and is partially supersonic. However, Endeve et al.'s focus was on turbulent magnetic field amplification and they employed an idealized setup that left out much of the essential CCSN microphysics. More work in 3D with high-resolution full-physics simulations will be needed to fully characterize SASI-induced turbulence. 


\section{Pre-Supernova Turbulence}

\subsection{Turbulent Convection in Advanced Burning Stages}

Most CCSN simulations start at the onset of the collapse of perfectly sphericallysymmetric cores, computed using 1D stellar evolution codes. These employ effective treatments of convective mixing and overshooting, e.g., [23, 24, 99, that can account for some of the effects of convection, especially over secular timescales. However there are still important uncertainties associated with the use of these prescriptions, partly because turbulent convection in stellar interiors is still not completely understood, see e.g., 65, 100 109, for some recent works.

A more immediate concern for CCSN modelers is the fact that one-dimensional stellar evolution codes cannot predict the properties of non-spherical perturbations of the progenitor present at the onset of collapse. Convective velocities during advanced burning stages immediately prior to collapse could reach hundreds of kilometers per second [104, 107, 109]. Convection, as well as gravity waves excited in the iron core during silicon shell burning [110, will be frozen in the collapsing core after the onset of collapse. They will be amplified as they cross the shock (Sec. 4.2), and they may play an important role in triggering explosions (Sec. 5).

\subsection{The Growth of Seed Perturbations in the Accretion Flow}

In the process of stellar core-collapse, the convective flow in nuclear burning shells undergoes profound evolution during its journey towards the shock. In the absence of nuclear burning and dissipative processes, the specific entropy of the flow is conserved, while the convective velocities increase due to conservation of angular momentum. If present, nuclear burning should in principle amplify convection, although the degree of amplification is limited if the collapse timescale is shorter than the convective turnover timescale. The dissipative processes should do the opposite. The vorticity waves, which have solenoidal velocity field, perturb the isodensity contours of the collapsing star, leading to the emission of powerful pressure waves 63. Entropy fluctuations also generate pressure waves as they accrete in a converging flow (e.g, 111 113]).

The evolution of hydrodynamic perturbations in accretion flows has been studied in a number of works (e.g., [111, 113-117). A general convective flow can be decomposed into the three physical modes: acoustic, vorticity, and entropy waves (e.g., [118]). Kovalenko \& Eremin [111] studied the evolution of all these three modes for Bondi accretion. Using linearized hydrodynamics equations, they obtained simple analytical relations in the limit $r \rightarrow 0$ for the scaling of perturbation amplitudes with radius. In particular, for $\ell>0$ acoustic and vorticity modes in the regime of supersonic mean flow, they find

$$
\begin{aligned}
\frac{\delta \rho}{\rho} & \propto 2 \ell(\ell+1) r^{-1 / 2}, \\
\frac{\delta v_{r}}{v_{r}} & \propto \ell(\ell+1) r^{(4-3 \gamma) / 4}, \\
\frac{\delta v_{\perp}}{v_{r}} & \propto r^{-1 / 2} \\
\frac{\delta v_{\text {rot }}}{v_{r}} & \propto r^{-1 / 2}
\end{aligned}
$$


where $\gamma$ is the adiabatic index of the gas, $\rho$ and $v_{r}$ are unperturbed values of density and radial velocity, while $\delta \rho, v_{\perp}$, and $v_{\text {rot }}$ are the perturbations of density and angular components of velocity, respectively. Lai \& Goldreich [114 found similar relations for perturbations in collapsing clouds in the same $r \rightarrow 0$ limit.

Takahashi \& Yamada [116] performed an analysis of the evolution of infalling perturbations in spherical supersonic Bondi accretion without imposing the $r \rightarrow 0$ limit and obtained the following scaling relations

$$
\begin{aligned}
\frac{\delta \rho}{\rho} & \propto \ell r^{-(5-3 \gamma) / 4} \\
\frac{\delta v_{r}}{v_{r}} & \propto \ell r^{(5-3 \gamma) / 4} \\
\frac{\delta v_{\perp}}{v_{r}} & \propto \text { const }, \\
\frac{\delta v_{\text {rot }}}{v_{r}} & \propto \ell r^{-1 / 2}
\end{aligned}
$$

These are somewhat different from corresponding scaling relations in [111, 114] and parts of the differences stem from the $r \rightarrow 0$ limit imposed in these works. A common limitation shared by these works is that they either consider only the supersonic region of accretion [114, 116] or only either supersonic or subsonic solutions of the Bondi accretion [111. In CCSNe, the convective Si/O burning shells initially infall subsonically, but then gradually accelerate and become supersonic. A detailed analysis of the evolution of convective perturbations from the subsonic to the supersonic regime, including the effects of possible nuclear burning and turbulent dissipation, is currently lacking in the literature.

Müller et al. 117] performed supernova simulations of a $3 \mathrm{D} 18 M_{\odot}$ progenitor model. They observed that, prior to collapse, the convective motion in the $\mathrm{O}$ shell primarily of solenoidal velocity field, while the density perturbations remain small. However, by the time the $\mathrm{O}$ shell reaches the shock, they observed strong density perturbations with amplitude $\delta \rho / \rho \simeq 0.1$ and peak angular wavenumber of $\ell \simeq 2$. They found that the spatial spectrum of the density perturbations prior to shockcrossing is very similar to that of the turbulent Mach number in the $\mathrm{O}$ shell prior to collapse. More specifically, for the normalized time-averaged spectrum of density perturbation, they found

$$
\frac{\hat{\rho}_{\ell}}{\hat{\rho_{0}}} \simeq 0.5 \hat{\mathcal{M}}_{r, \ell},
$$

where $\hat{\mathcal{M}}_{r, \ell}$ is the spectrum of the Mach number of radial velocity in the middle of the O shell (see their equation 6). Such as simple relation between the spectrum of the velocity perturbation in the oxygen shell and that of density perturbations just ahead of the shock has not been established by the aforementioned studies of the evolution of perturbations in Bondi accretion. This discrepancy may stem from the fact that the perturbative studies consider only the supersonic regime of collapse and use stationary Bondi flow, which neglects the details of progenitor density structures (see [117] for in-depth discussion). 


\section{Turbulence Shock Interaction}

\subsection{Numerical Results}

Couch \& Ott 21 were the first to show the impact of the accretion of perturbations in simulations. They considered the $15-M_{\odot}$ progenitor from Woosley \& Heger [119, which they evolved in 3D. They performed simulations either with the original, unperturbed, progenitor structure, or with velocity perturbations. They found that the accretion of perturbations enhances the turbulent kinetic energy in the gain region, especially at large scales. This has a number of beneficial effects for the explosion, as discussed in Sec. 2.2, and, in the case of the simulations of [21, it was sufficient to trigger an explosion in an otherwise failing model.

Müller \& Janka 63 further explored the impact of perturbations in the accretion flow in 2D axisymmetric simulations. They systematically studied the effects of the character and angular scale of the perturbations. They found that in the case of velocity perturbations, low-order modes $(\ell=1$ or $\ell=2)$ are the most effective in triggering explosions. Müller \& Janka [63] quantified the impact of perturbations in terms of the critical neutrino luminosity and found a decrease of up to $10 \%$ for large-scale velocity perturbations. Density perturbations were also found to be very effective. However, a significant impact was found only for large perturbations, corresponding to pre-collapse turbulent Mach numbers of $\gtrsim 0.3$, which might be present only in a small fraction of progenitors [109].

In their analysis of the effects of perturbations on the post-shock turbulence, Müller \& Janka 63 emphasized the role of the directionally-dependent modulation of the accretion rate ahead of the shock. However, analytic studies (120; Sec. 5.2) have shown that the effects of shock-turbulence interaction in CCSNe have a rather complex phenomenology and that their effectiveness depends on the relative phase of entropy and vorticity perturbations accreted through the shock. In any case, both simulations and analytic theory suggest that the effect of perturbations depends on their detailed nature. This motivates the development of well motivated perturbed progenitor models.

Chatzopoulos et al. 121 developed a systematic method to characterize precollapse asphericities in CCSN progenitors. They proposed a method to extract characteristic properties of convection from multi-dimensional simulations of advanced burning stages in a way that allows them to be injected in otherwise spherical progenitor models at the verge of core collapse. More recently, Couch et al. 104. and Müller et al. 107, 117] simulated, for the first time, CCSN progenitors that were self-consistently evolved in multi-D in the last minutes prior to collapse.

Couch et al. [104] studied a $15-M_{\odot}$ progenitor. They self-consistently simulated the last few minutes of Si-burning, the collapse of the iron core, and the subsequent core bounce and explosion. They used a reduced 21-isotope nuclear network and a neutrino leakage scheme with parametrized heating. As a baseline for comparison, they re-simulated the collapse, bounce, and subsequent evolution of the same ironcore after angle averaging. They found that (non-spherical) turbulent fluctuations produced during Si burning resulted in an earlier and more energetic explosion. A possible caveat of this first study, however, is that Si burning was simulated only in an octant (assuming reflection symmetry across the coordinate planes). This prevented the appearance of convective cells with large angular scale, which seem to be the most effective for triggering explosions 63 . Consequently, the effects of perturbations 
might be even larger than found by Couch et al. 104.

Müller et al. [107, 117] considered instead an 18- $M_{\odot}$ progenitor. They simulated the last $\sim 5$ minutes of evolution of the oxygen-burning shell in $4 \pi$ (but excised most of the $\mathrm{Fe} / \mathrm{Si}$ core of the star) 107. The resulting progenitor was subsequently evolved in 3D with their fast multi-group transport method 63. They found that perturbations determined the outcome of core collapse: while the progenitor with perturbations exploded $\sim 0.3$ seconds after bounce, the spherically-averaged progenitor did not explode within the end of their simulation ( 0.625 seconds after bounce). A third progenitor, obtained with an artificially reduced burning rate resulting in smaller convective velocities prior to collapse, exploded at $\sim 0.5$ seconds after bounce.

\subsection{Analytical Results}

5.2.1. Linear Analysis of Shock-Turbulence Interaction. Abdikamalov et al. 120] studied the impact of pre-shock perturbations on the evolution of the shock and postshock flow using a linear perturbation theory known as the linear interaction analysis (LIA) (e.g., 122]). In the LIA, the flow is decomposed into mean and fluctuating parts. The unperturbed shock is assumed to be planar and the mean flow is assumed to be uniform in space. While these are approximations, the strength of the LIA lies it its simplicity, which allows one to obtain deep insight into the physics of shockturbulence interaction (e.g., 122]). Furthermore, in its regime of applicability, when the turbulent Mach number of the perturbations ahead of the shock is small, the LIA shows excellent agreement with high-resolution numerical simulations [123.

In the LIA, the mean flow obeys the usual Rankine-Hugoniot conditions, while the fluctuations obey the linearized version of the jump conditions. The upstream flow completely determines the downstream flow via these conditions (e.g., [124 128]). The perturbations can be decomposed into vorticity, entropy, and acoustic modes, each of which evolve independently at the linear order [118. With this decomposition, the LIA formalism can be applied to each mode individually. The results can then be integrated to get the full depiction of shock-turbulence interaction to linear order.

Abdikamalov et al. 120 considered incoming field of vorticity and entropy waves. When vorticity and/or entropy waves hit a shock, they generate a downstream field of vorticity, entropy, and acoustic waves. Abdikamalov et al. 120 showed that the kinetic energy of fluctuations in the post-shock region is almost entirely due to vorticity waves, with acoustic waves contributing only $\lesssim 2 \%$ of the total kinetic energy. They found that the total kinetic energy of turbulent fluctuations is amplified by a factory of $\sim 2$ at shock crossing. The angular component is amplified by a factor of $\sim 3$, while the radial component does not undergo significant amplification (cf. Fig 7).

5.2.2. Impact on the Explosion Condition The impact of the progenitor asphericities on the explosion condition has been estimated analytically using the concept of the critical (i.e., mininum) neutrino luminosity necessary to drive an explosion [63, 107, 120; but, see: Nagakura et al. 130] for a possible alternative approach. In the absence of non-radial motion in the gain region, the critical luminosity can be estimated as 131]

$$
\left(L_{\nu} E_{\nu}^{2}\right)_{\text {crit }} \propto(\dot{M} M)^{3 / 5} r_{\text {gain }}^{-2 / 5},
$$

which is approximately equivalent to condition $\tau_{\text {adv }} / \tau_{\text {heat }} \gtrsim 1$, where $\tau_{\text {adv }}$ and $\tau_{\text {heat }}$ are the timescales of advection and heating in the gain region, and $r_{\text {gain }}$ is the radius 


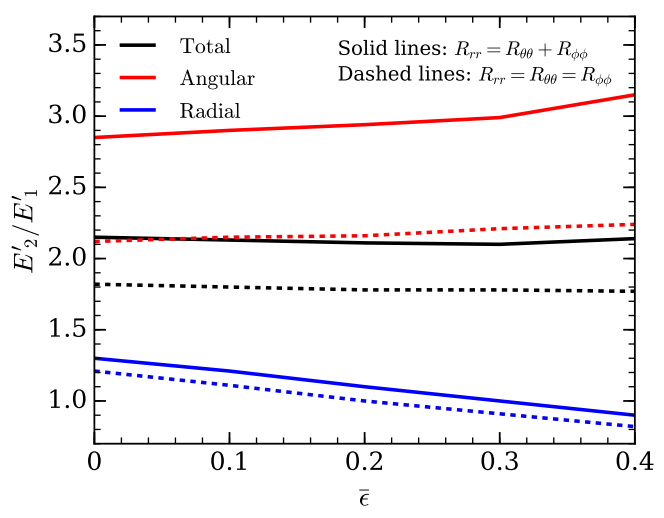

Figure 7. The amplification of turbulent kinetic energy at shock crossing for incoming incident waves as a function of dimensionless nuclear dissociation parameter. The latter characterizes the strength of nuclear dissociation at the shock, larger values of $\bar{\epsilon}$ corresponding to stronger dissociation [56 129. The black lines show the amplification of the total kinetic energy, while blue and red lines show the amplifications of radial and angular components of kinetic energy. The solid lines represent anisotropic turbulence characterized by the relation $R_{r r}=R_{\theta \theta}+R_{\phi \phi}$, while the dashed lines represent fully isotropic turbulence. The Mach number of the pre-shock mean flow is assumed to be 5 . Reprinted from E. Abdikamalov, A. Zhaksylykov, D. Radice, and S. Berdibek, Shockturbulence interaction in core-collapse supernovae, Monthly Notices of the Royal Astronomical Society 461, 3864 (2016) [120, (C) E. Abdikamalov, A. Zhaksylykov, D. Radice, and S. Berdibek. Reproduced with permission.

at which the net neutrino heating becomes positive [131. The effect of post-shock turbulence can be included as an isotropic pressure contribution (cf. Eq. 10), which leads to a lower critical luminosity [63]:

$$
\left(L_{\nu} E_{\nu}^{2}\right)_{\text {crit }} \propto(\dot{M} M)^{3 / 5} r_{\text {gain }}^{-2 / 5}\left(1+\frac{4\left\langle\mathcal{M}_{2}^{\prime 2}\right\rangle}{3}\right)^{-3 / 5},
$$

where $\left\langle\mathcal{M}_{2}^{\prime 2}\right\rangle$ is the average Mach number of the turbulent flow in the post-shock region. Pre-shock fluctuations cross the shock and contribute to $\mathcal{M}_{2}^{\prime}$ in the postshock region. The reduction of the critical luminosity due to this "direct injection" of kinetic energy can be estimated as 120]

$$
\frac{\Delta L_{\mathrm{crit}}}{L_{\mathrm{crit}}} \simeq \frac{4}{5} \frac{E_{\mathrm{a}, 2}^{\prime}}{E_{\mathrm{a}, 1}^{\prime}} \frac{\left\langle c_{\mathrm{s}, 1}^{2}\right\rangle}{\left\langle c_{\mathrm{s}, 2}^{2}\right\rangle}\left\langle\mathcal{M}_{1}^{\prime 2}\right\rangle,
$$

where $\left\langle c_{\mathrm{s}, 1}^{2}\right\rangle$ and $\left\langle c_{\mathrm{s}, 2}^{2}\right\rangle$ are the sound speeds in the pre-shock and post-shock regions. In the absence of turbulent dissipation, the ratio $E_{\mathrm{a}, 2}^{\prime} / E_{\mathrm{a}, 1}^{\prime}$ of the turbulent kinetic energies in the immediate post-shock and pre-shock regions can be obtained from the LIA formalism [120]. For typical parameters of the accretion shock, and assuming anisotropic turbulence with $R_{r r}=R_{\theta \theta}+R_{\phi \phi}$, Eq. (30) yields

$$
\frac{\Delta L_{\text {crit }}}{L_{\text {crit }}} \simeq 0.6\left\langle\mathcal{M}^{\prime 2}{ }_{1}^{2},\right.
$$

The turbulent Mach number in nuclear burning shells is $~ 0.1$ (e.g., 107, 109]). The conservation of angular momentum dictates that, in the absence of turbulent 
dissipation and nuclear burning, the Mach number of non-radial fluctuations should increase as $\propto r^{(3 \gamma-7) / 4}$ during accretion [111, 114]. Accordingly, if convective shells accrete from $\sim 1500 \mathrm{~km}$ toward the shock at $\sim 200 \mathrm{~km}$, the turbulent Mach number increases by a factor of $\sim 4.5$ to $\sim 0.45$. Compared to the case with no pre-collapse perturbations, this leads to a reduction of the critical luminosity by $\sim 12 \%$.

Recent 3D neutrino-hydrodynamics simulations of the $18 M_{\odot}$ progenitor model infer a reduction of $\sim 20 \%$ in the critical luminosity [117, which is significantly larger than the above estimate. This suggests that the direct injection of kinetic energy plays a sub-dominant role in triggering an explosion 63. An additional contribution can come from buoyant driving of convection in the post-shock region.

An alternative approach that approximately includes this effect was presented by [107. Their calculation is based on the observation that pre-collapse perturbations with Mach number $\mathcal{M}$ generate density perturbations with relative amplitude of $\sim \mathcal{M}$ by the time they reach the shock. After crossing the shock, these density perturbations become buoyant and seed turbulent convection in the gain region. The reduction of the critical luminosity can be estimated as 107.

$$
\frac{\Delta L_{\text {crit }}}{L_{\text {crit }}} \simeq 0.47 \frac{\mathcal{M}}{\ell \eta_{\text {acc }} \eta_{\text {heat }}},
$$

where $\ell$ is the peak angular wave number of the perturbations, $\eta_{\text {heat }}$ is the neutrinoheating efficiency, $\eta_{\text {acc }}$ is the efficiency of conversion of accretion energy into electronflavor neutrino emission. Recently, Huete et al. [128] pointed out the importance of entropy perturbations generated by the interaction of the supernova shock with vorticity waves originating from nuclear burning shells. These perturbations are associated with density fluctuations, which become buoyant and contribute to the turbulent convection in the gain region. Depending on problem parameters, the resulting reduction in the critical luminosity was estimated to be

$$
\frac{\Delta L_{\text {crit }}}{L_{\text {crit }}} \simeq(0.68-0.96) \times \frac{\mathcal{M}}{\ell \eta_{\text {acc }} \eta_{\text {heat }}} .
$$

For typical values of $\eta_{\text {heat }} \simeq 0.1$, and $\eta_{\text {acc }} \simeq 2$, this implies a $\sim 17-24 \%$ reduction of the critical luminosity for $\mathcal{M} \simeq 0.1$ and $\ell=2$. This is roughly in line with the results of $3 \mathrm{D}$ neutrino-hydrodynamics simulations of 117 .

5.2.3. Normal Mode Analysis. Takahashi et al. [132] studied the influence of upstream perturbations on convective and SASI modes. Their model is based on the solution of linearized hydrodynamic equations in the post-shock region with a boundary condition at the shock that mimics pre-shock perturbations. They considered a stationary spherically symmetric background model with neutrino heating. They applied perturbations of the specific form

$$
\begin{aligned}
& \frac{\delta \rho}{\rho}=\sin \left(\omega_{\mathrm{up}} t+\phi\right), \\
& \frac{\delta v}{v_{r}}=-0.5 \sin \left(\omega_{\mathrm{up}} t+\phi\right), \\
& \frac{\delta \epsilon}{\epsilon}=\sin \left(\omega_{\mathrm{up}} t+\phi\right),
\end{aligned}
$$

Here, $\omega_{\text {up }}$ is the frequency, $\phi$ is the phase of the perturbation, and $\epsilon$ is the specific internal energy. The linearized equations for the perturbation field are then solved using the Laplace transform, which allowed them to calculate the frequencies 


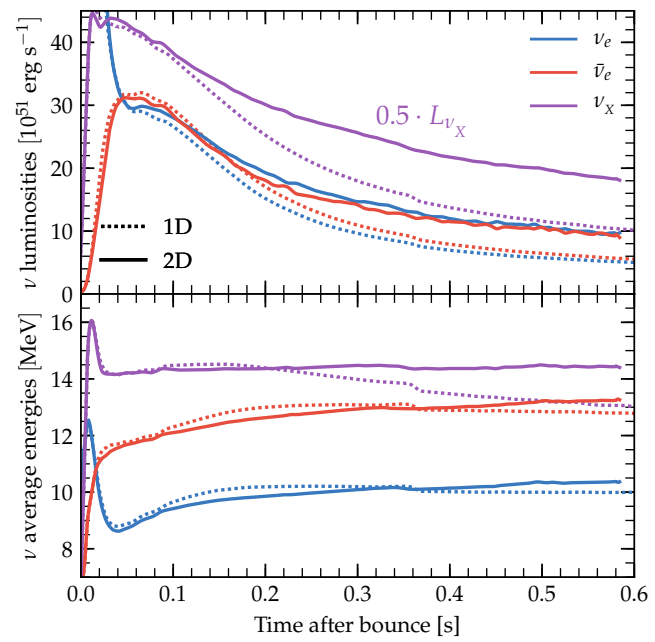

Figure 8. Neutrino luminosity (top panel) and average energies (bottom panel) at $10,000 \mathrm{~km}$ as a function of time for the $\mathrm{ONeMg}$ electron-capture supernova progenitor of Nomoto [137, 138, computed by Radice et al. 8] with their "Baseline" setup. Here, " $\nu_{X}$ " denotes the sum of all the heavy-lepton neutrinos. Since this progenitor model explodes in $1 \mathrm{D}$ and $2 \mathrm{D}$, the neutrino luminosities in both cases are only due to the cooling PNS and there is no contribution from the accretion luminosity. Consequently, the differences between $1 \mathrm{D}$ and $2 \mathrm{D}$ are attributable to the effect of PNS convection.

and growth rates of the SASI and convection modes under the influence of these perturbations. Takahashi et al. 132 systematically studied the dependence on frequency and phase of the perturbations and showed that resonant amplification of SASI and convection can occur when both the frequency and the growth rate of upstream perturbations match those of the intrinsic SASI and convective modes, respectively. Due to this rather stringent requirement, such resonances are unlikely to occur in CCSNe. However, they found that the amplitudes of intrinsic modes become larger by a factor of $\lesssim 10$ when the frequency of the upstream perturbations is close to that of the intrinsic SASI mode or to the growth rate of convection. This suggests that accreted perturbations might efficiently drive SASI and/or convection to nonlinear amplitudes.

\section{Protoneutron Star Convection}

The gain region is not the only area with convective overturn active after core bounce. Immediately after neutrino shock breakout, the weakening shock leaves behind a negative entropy gradient, which rapidly develops convection [51, 133 135. However, this initial convective overturn subsides within $\sim 10 \mathrm{~ms}$ [136], as the unstable entropy gradient is erased by neutrino heating and convective mixing.

Later, neutrino losses from the outer layers of the PNS establish negative entropy and lepton number gradients at densities above $\sim 10^{13} \mathrm{~g} \mathrm{~cm}^{-3}$. A second, much longer, period of convective overturn develops because of the presence of these gradients [12, 51, 136, 139 141]. The region of the PNS that is unstable to convection depends on the nuclear EOS, which can be seen from the thermodynamic derivatives 
appearing in the Brunt-Väisälä frequency (see Equation 1). In general, $(\partial p / \partial s)_{\rho, Y_{e}}$ is positive, so negative entropy gradients drive instability. On the other hand, $\left(\partial p / \partial Y_{e}\right)_{\rho, s}$ can be negative under the conditions encountered in the outer layers of the PNS. Here, the pressure is mainly determined by high-density, interacting neutron gas and the degenerate electron gas. If the neutron gas provides the dominant contribution, reducing the electron fraction increases the pressure. This implies the negative lepton gradients in the outer layers of the PNS can have a stabilizing influence for some EOSs and the convectively unstable region will depend on the nuclear EOS. This second phase of PNS convection can persist for many seconds and leave a detectable signature in the neutrino signal from the cooling PNS [142].

Early 2D calculations with energy-integrated neutrino transport [136] suggested that the convective transport of energy and lepton number from the PNS core toward the neutrinospheres could play an important role in the dynamics of the explosion, since it resulted in an increase of the neutrino luminosities by up to a factor two. However, the first 2D simulations with energy-dependent neutrino transport found a very different picture [140, 141. In these models, PNS convection was buried deep below the neutrinospheres by large stabilizing entropy gradients interior and exterior to it. Accordingly, PNS convection could only provide a $\sim 15 \%$ to $\sim 30 \%$ increase of the neutrino luminosities in the first $300 \mathrm{~ms}$ after bounce [140, 141.

More recently, Radice et al. 8] pointed out that, over longer timescales, the contraction of the PNS proceeds to the point that its surface comes into contact with the PNS convection region. Afterward, they found differences in the neutrino luminosities as large as a factor two between their 1D and 2D calculations, which they attributed to PNS convection. Fig. 8 shows the neutrino luminosities and average energies from their simulation of the $8.8 M_{\odot}$ (zero-age main sequence) progenitor from Nomoto [137, 138. Since this progenitor explodes quickly ( $\sim 50$ milliseconds after bounce) even in 1D calculations, the differences in the neutrino luminosities are inescapably related to PNS convection and are not due to differences in the accretion flow. The figure demonstrates $\sim 2 \times$ boost of the luminosities, as well as a slight increase in the average neutrino energies. A similar enhancement of the heavy-lepton neutrino luminosities was also found by O'Connor \& Couch 27. However, they did not find a correspondingly large boost of the electron-type neutrino luminosity, presumably because the smaller luminosities in their $1 \mathrm{D}$ calculations were offset by the larger accretion luminosities.

Other consequences of PNS convection are a reduced contraction rate of the PNS [8, 140] and the launching of gravity waves in the stably stratified region above it [141]. The first effect is mostly negative for the prospects of the explosion, since it results in a reduction of the average neutrino energies by several percent. This reduction, in turn, reduces the coupling between the neutrinos and the matter behind the shock. The second effect is, instead, positive, since gravity waves transport energy out of the core and release it near the surface of the PNS. This energy is subsequently radiated as neutrinos and, in part, reabsorbed in the gain layer behind the shock.

The Ledoux-stable region above the inner convection layer received significant attention before the advent of multidimensional simulations with spectral neutrino transport. Wilson and Mayle [143, 144] suggested that a doubly diffusive instability might operate in this region. They argued that if a parcel of neutron rich matter is displaced toward the center of the PNS, then it would thermally equilibrate on a very short timescale. However, because of the net cancellation of the interaction with electron and anti-electron neutrinos, this material would still be more neutron 
rich than the surroundings. As a result of the smaller electron pressure, the fluid blob would keep on sinking. This process is called neutron fingering in analogy with the salt fingering observed in Earth's oceans [145. Convective overturn would then rapidly develop and transport lepton number and heat from the core of the PNS all the way to the neutrinospheres. This would significantly boost the neutrino luminosities. Indeed, their 1D models with an effective prescription of the neutron-finger instability successfully exploded [144.

However, Bruenn \& Dineva [146] later pointed out that a radially displaced fluid element below the neutrinospheres would reach equilibration mostly because of the emission of low energy electron-type neutrinos. The reason is, on the one hand, that heavy-lepton neutrinos are not effective at transporting heat and, on the other hand, that high energy electron-type neutrinos would be trapped due to their small mean free path. Consequently, the displaced fluid element would achieve lepton-number reequilibration before cooling down to the ambient temperature, and the neutron-finger instability should not develop. This has since been confirmed by multidimensional simulations, which found no evidences for the neutron finger or other doubly diffusive instabilities [147] in the PNS [140, 141].

\section{Magnetic Effects}

Magnetic fields can play an important role in the CCSN explosion mechanism. In rapidly rotating progenitors, they act as an agent powering jet-like outflows by extracting rotational energy, e.g., [148-150]. The necessary magnetar-strength field $\left(\sim 10^{15} \mathrm{G}\right)$ can be obtained via flux-compression and winding from strongly magnetized progenitor cores [151] or via magnetoturbulence [152 154] in the shear layer surrounding the PNS. While many studies have focused on magnetic fields in rapidly rotating progenitors, the impact of magnetic fields in slowly or non-rotating progenitors has so far received limited attention in the literature.

Endeve et al. 68, 155 investigated magnetic field amplification by the SASI in $2 \mathrm{D}$ axisymmetric and 3D simulations. They found that SASI-driven turbulence in the post-shock region amplifies magnetic field exponentially exponentially (i.e., by multiple orders of magnitude) and can account for neutron star magnetic field of $\sim 10^{14} \mathrm{G}$ or more in non-rotating progenitors. However, while the magnetic field amplification impacted the local flow properties in their simulations, they did not find a modification of the global dynamics.

Obergaulinger et al. [156] studied magnetic amplification and evolution of nonrotating magnetized CCSNe via $2 \mathrm{D}$ axisymmetric simulations. They considered a $15 M_{\odot}$-progenitor augmented with a seed magnetic fields, the strength of which they varied. They found that convection and the SASI amplify magnetic fields in the gain region up to a factor $\sim 5$, which they explained in terms of the ratio between the advection and the eddy-turnover times. As a consequence of this modest amplification factor their weakly magnetized models - with pre-collapse fields smaller than $10^{12} \mathrm{G}$ evolved very similarly to purely hydrodynamical models. Only in their $10^{12} \mathrm{G}$ model did the magnetic field energy reach equipartition with the kinetic energy, thereby enabling it to trigger a considerably earlier explosion.

Summarizing, extant studies on magnetized CCSNe are inconclusive concerning the role that magnetic fields can play in the explosion of slowly- or non-rotating progenitors. On the one hand, global qualitative changes in the dynamics have been reported only for very large pre-collapse fields. On the other hand, a quantitative 
impact on the flow is present even for realistic seed fields. Moreover, the simulations that have been carried out so far only establish a lower bound for the amplification factor of the magnetic fields. Axisymmetric simulations significantly restrict the possibilities for magnetic field amplification, as illustrated by the anti-dynamo theorem [157], see also [158]. In addition, 3D studies are limited in resolution and may have not reached sufficiently high effective Reynolds numbers to fully capture the magnetic field amplification. Future studies that investigate magnetic field amplification and their potential to alter the flow properties in $3 \mathrm{D}$ are needed to understand their role and impact for slowly or non-rotating CCSN progenitors.

\section{Conclusions}

Turbulence is an important component of the CCSN explosion mechanism. Turbulent convection during the last stages of nuclear burning creates perturbations that are then frozen in the collapsing gas after the unset of core collapse. These perturbations can seed fluid instabilities, such as the SASI or neutrino-driven convection, as they are accreted by the shock. The development of strong SASI and/or neutrino-driven convection, in turn, creates favorable conditions for the explosion. Convection inside the PNS can boost the neutrino luminosity and might in some cases contribute to shock revival. Finally, magnetic fields are expected to be amplified by convection and/or by other MHD instabilities, such as the magnetorotational instability (MRI), and might play an important role in the explosion.

Turbulence may also be important in understanding and interpreting observations of CCSNe. This is particularly true for CCSN remnants such as Cassiopeia A [159]. In Cas A, recent observations suggest the presence of radioactive nickel-heated bubbles expanding in the remnant, the structure of which may be reflective of the turbulence in the explosion mechanism itself [160. Additionally, the presence of high-velocity knots at large radius in the remnant could also be the result of turbulent structures in the mechanism that have become ballistic within the expanding remnant [161]. Observational evidence for turbulence in the CCSN mechanism also comes from complex spectral features in several objects. In the case of SN 1987A, detailed analysis of He I lines indicates the turbulent mixing of radioactive nickel to large velocities [162]. The so-called "Bochum" event in SN 1987A [163] may also be evidence for turbulent mixing in the explosion [164. Multiply peaked O lines in various stripped envelope CCSNe entering the nebular phase may also imply the action of turbulence in the inner workings of the CCSN mechanism [165].

Many of these important parts of the CCSN explosion mechanism puzzle have been pieced together in the past few years. However, this progress has been driven either by specialized/idealized simulations that addressed only one of these aspects in isolation, or by means of simulations that ostensibly included all of the relevant physics, but lacked the numerical resolution necessary to capture all scales of the problem. Solving the CCSN explosion mechanism problem will necessarily require a way to bridge the gap between these two approaches. A possible way forward might be the development of subgrid-scale models for the effective treatment of turbulence in global simulations. This will be an object of our future work. 


\section{Acknowledgments}

The authors acknowledge Adam Burrows, Jérôme Guilet, Roland Haas, Thierry Foglizzo, Bernhard Müller, Jeremiah W. Murphy, and Evan O'Connor for insightful discussion on the explosion mechanism of core-collapse supernovae, and the anonymous referee for valuable suggestions that improved the quality of this manuscript. DR gratefully acknowledges support from the Schmidt Fellowship, the Sherman Fairchild Foundation and the Max-Planck/Princeton Center (MPPC) for Plasma Physics (NSF PHY-1523261). CDO is partially supported by NSF grant CAREER PHY1151197. SMC is supported by the U.S. Department of Energy, Office of Science, Office of Nuclear Physics, under Award Numbers de-sc0015904 and de-sc0017955 and the Chandra X-ray Observatory under grant TM7-18005X. Work presented in this review benefitted from computer time allocations at NSF/NCSA Blue Waters (PRAC ACI-1440083), at the National Energy Research Scientific Computing Center (project m152), a DOE Office of Science User Facility supported by the Office of Science of the U.S. Department of Energy under Contract No. DE-AC02-05CH11231, and on the Texas Advanced Computing Center Stampede cluster under NSF XSEDE allocation TG-PHY100033. Simulations described herein where completed with computer time provided by the Innovative and Novel Computational Impact on Theory and Experiment (INCITE) program. This research used resources of the Argonne Leadership Computing Facility, which is a DOE Office of Science User Facility supported under Contract DE-AC02-06CH11357.

\section{References}

[1] Colgate S A and McKee C 1969 Astrophys. J. 157623

[2] Bethe H A and Wilson J R 1985 Astrophys. J. 295 14-23

[3] Rampp M and Janka H T 2000 Astrophys. J. Lett. 539 L33

[4] Liebendörfer M, Mezzacappa A, Thielemann F K, Messer O E B, Hix W R and Bruenn S W 2001 Phys. Rev. D 63103004

[5] Thompson T A, Burrows A and Pinto P A 2003 Astrophys. J. 592434

[6] Liebendörfer M, Rampp M, Janka H T and Mezzacappa A 2005 Astrophys. J. 620840

[7] Sumiyoshi K, Yamada S, Suzuki H, Shen H, Chiba S and Toki H 2005 Astrophys. J. 629922

[8] Radice D, Burrows A, Vartanyan D, Skinner M A and Dolence J C 2017 Astrophys. J. 85043

[9] Blondin J M, Mezzacappa A and DeMarino C 2003 Astrophys. J. 584971

[10] Foglizzo T, Galletti P, Scheck L and Janka H T 2007 Astrophys. J. 6541006

[11] Herant M 1995 Phys. Rep. 256117

[12] Burrows A, Hayes J and Fryxell B A 1995 Astrophys. J. 450830

[13] Janka H T and Müller E 1996 Astron. and Astrophys. 306167

[14] Foglizzo T, Scheck L and Janka H T 2006 Astrophys. J. 6521436

[15] Abdikamalov E, Ott C D, Radice D, Roberts L F, Haas R, Reisswig C, Mösta P, Klion H and Schnetter E 2015 Astrophys. J. 80870

[16] Garnier E, Adams N and Sagaut P 2000 Large Eddy Simulation for Compressible Flows (Springer, Berlin, Germany)

[17] Aubard G, Stefanin Volpiani P, Gloerfelt X and Robinet J C 2013 Flow, Turbulence and Combustion 91 497-518

[18] Radice D, Ott C D, Abdikamalov E, Couch S M, Haas R and Schnetter E 2016 Astrophys. J. 82076

[19] Kraichnan R H 1967 Phys. Fluids 101417

[20] Lentz E J, Bruenn S W, Hix W R, Mezzacappa A, Messer O E B, Endeve E, Blondin J M, Harris J A, Marronetti P and Yakunin K N 2015 Astrophys. J. Lett. 807 L31

[21] Couch S M and Ott C D 2015 Astrophys. J. 7995

[22] Couch S M and O'Connor E P 2014 Astrophys. J. 785123

[23] Paxton B, Bildsten L, Dotter A, Herwig F, Lesaffre P and Timmes F 2011 Astrophys. J. Supp. 1923 
[24] Paxton B, Cantiello M, Arras P, Bildsten L, Brown E F, Dotter A, Mankovich C, Montgomery M H, Stello D, Timmes F X and Townsend R 2013 Astrophys. J. Supp. 2084

[25] Fryxell B, Olson K, Ricker P, Timmes F X, Zingale M, Lamb D Q, MacNeice P, Rosner R, Truran J W and Tufo H 2000 Astrophys. J. Supp. 131273

[26] Dubey A, Reid L B, Weide K, Antypas K, Ganapathy M K, Riley K, Sheeler D and Siegal A 2009 Parallel Comput. 35512

[27] O'Connor E and Couch S M 2015 submitted to Astrophys. J.;arXiv:1511.07443

[28] Vincent A and Meneguzzi M 1991 Journal of Fluid Mechanics 2251

[29] Janka H T, Hanke F, Hüdepohl L, Marek A, Müller B and Obergaulinger M 2012 Prog. Th. Exp. Phys. 2012 01A309

[30] Burrows A 2013 Rev. Mod. Phys. 85245

[31] Janka H T, Melson T and Summa A 2016 Ann. Rev. Nuc. Part. Sc. 66341

[32] Müller B 2016 Pub. Astron. Soc. Aus. 33 e048

[33] Chandrasekhar S 1939 An Introduction to the Study of Stellar Structure

[34] Ledoux P 1947 Astrophys. J. 105305

[35] Scheck L, Janka H T, Foglizzo T and Kifonidis K 2008 Astron. and Astrophys. 477931

[36] Burrows A, Dolence J C and Murphy J W 2012 Astrophys. J. 7595

[37] Hanke F, Marek A, Müller B and Janka H T 2012 Astrophys. J. 755138

[38] Hanke F, Müller B, Wongwathanarat A, Marek A and Janka H T 2013 Astrophys. J. 77066

[39] Fernández R, Müller B, Foglizzo T and Janka H T 2014 Mon. Not. Roy. Astron. Soc. 4402763

[40] Iwakami W, Nagakura H and Yamada S 2014 Astrophys. J. 786118

[41] Fernández R 2015 Mon. Not. Roy. Astron. Soc. 452 2071-2086

[42] Cardall C Y and Budiardja R D 2015 Astrophys. J. Lett. 813 L6

[43] Ott C D, Abdikamalov E, Mösta P, Haas R, Drasco S, O'Connor E P, Reisswig C, Meakin C A and Schnetter E 2013 Astrophys. J. 768115

[44] Dolence J C, Burrows A, Murphy J W and Nordhaus J 2013 Astrophys. J. 765110

[45] Murphy J W, Dolence J C and Burrows A 2013 Astrophys. J. 77152

[46] Couch S M 2013 Astrophys. J. 77535

[47] Takiwaki T, Kotake K and Suwa Y 2014 Astrophys. J. 78683

[48] Melson T, Janka H T and Marek A 2015 Astrophys. J. Lett. 801 L24

[49] Melson T, Janka H T, Bollig R, Hanke F, Marek A and Müller B 2015 Astrophys. J. Lett. 808 L42

[50] Roberts L F, Ott C D, Haas R, O'Connor E P, Diener P and Schnetter E 2016 Astrophys. J. 83198

[51] Bethe H A 1990 Rev. Mod. Phys. 62801

[52] Herant M, Benz W, Hix W R, Fryer C L and Colgate S A 1994 Astrophys. J. 435339

[53] Mezzacappa A, Calder A C, Bruenn S W, Blondin J M, Guidry M W, Strayer M R and Umar A S 1998 Astrophys. J. 495911

[54] Burrows A and Goshy J 1993 Astrophys. J. Lett. 416 L75

[55] Murphy J W, Ott C D and Burrows A 2009 Astrophys. J. 7071173

[56] Fernández R and Thompson C 2009 Astrophys. J. 7031464

[57] Marek A and Janka H T 2009 Astrophys. J. 694664

[58] Fernández R 2012 Astrophys. J. 749142

[59] Müller B, Janka H T and Marek A 2012 Astrophys. J. 75684

[60] Yamasaki T and Yamada S 2007 Astrophys. J. 6561019

[61] Murphy J W and Burrows A 2008 Astrophys. J. 6881159

[62] Murphy J W and Meakin C 2011 Astrophys. J. 74274

[63] Müller B and Janka H T 2015 Mon. Not. Roy. Astron. Soc. 448 2141-2174

[64] Radice D, Couch S M and Ott C D 2015 Comp. Astrophy. Cosmol. 27

[65] Arnett D, Meakin C and Young P A 2009 Astrophys. J. 6901715

[66] Mabanta Q and Murphy J W 2017 ArXiv e-prints: 1706.0007

[67] Murphy J W and Dolence J C 2017 Astrophys. J. 834183

[68] Endeve E, Cardall C Y, Budiardja R D, Beck S W, Bejnood A, Toedte R J, Mezzacappa A and Blondin J M 2012 Astrophys. J. 75126

[69] Handy T, Plewa T and Odrzywolek A 2014 Astrophys. J. 783125

[70] Frisch U 1996 Turbulence: The Legacy of A. N. Kolmogorov (Cambridge University Press)

[71] Peebles P J E 1993 Principles of Physical Cosmology (Princeton Univ. Press, Princeton, NJ, USA)

[72] Couch S M and Ott C D 2013 Astrophys. J. Lett. 778 L7

[73] Müller B and Janka H T 2014 Astrophys. J. 78882

[74] Porter D H, Woodward P R and Pouquet A 1998 Phys. Fluids 10237 
[75] Benzi R, Biferale L, Fisher R T, Kadanoff L P, Lamb D Q and Toschi F 2008 Phys. Rev. Lett. 100234503

[76] Goldreich P and Keeley D A 1977 Astrophys. J. 211 934-942

[77] Summa A, Janka H T, Melson T and Marek A 2017 accepted for publication in Astrophys. J.; arXiv:1708.04154

[78] Sytine I V, Porter D H, Woodward P R, Hodson S W and Winkler K H 2000 J. Comp. Phys. 158225

[79] Yakhot V and Zakharov V 1993 Phys. D 64379

[80] She Z and Jackson E 1993 Phys. Fluids 51526

[81] Falkovich G 1994 Phys. Fluids 6 1411-1414

[82] Verma M K and Donzis D 2007 J. Phys. A 404401

[83] Frisch U, Kurien S, Pandit R, Pauls W, Ray S, Wirth A and Zhu J Z 2008 Phys. Rev. Lett. 101144501

[84] Blondin J M and Mezzacappa A 2007 Nature 44558

[85] Iwakami W, Kotake K, Ohnishi N, Yamada S and Sawada K 2008 Astrophys. J. 6781207

[86] Fernández R 2010 Astrophys. J. 7251563

[87] Iwakami W, Kotake K, Ohnishi N, Yamada S and Sawada K 2009 Astrophys. J. 700232

[88] Nakamura K, Kuroda T, Takiwaki T and Kotake K 2014 Astrophys. J. 79345

[89] Blondin J M, Gipson E, Harris S and Mezzacappa A 2017 Astrophys. J. 835170

[90] Foglizzo T 2009 Astrophys. J. 694820

[91] Sato J, Foglizzo T and Fromang S 2009 Astrophys. J. 694833

[92] Guilet J and Foglizzo T 2012 Mon. Not. Roy. Astron. Soc. 421546

[93] Blondin J M and Mezzacappa A 2006 Astrophys. J. 642401

[94] Laming J M 2007 Astrophys. J. 6591449

[95] Burrows A, Livne E, Dessart L, Ott C D and Murphy J 2006 Astrophys. J. 640878

[96] Guilet J, Sato J and Foglizzo T 2010 Astrophys. J. 7131350

[97] Burrows A, Livne E, Dessart L, Ott C D and Murphy J 2007 Astrophys. J. 655416

[98] Müller B, Janka H T and Heger A 2012 Astrophys. J. 76172

[99] Cox J P and Giuli R T 1968 Principles of Stellar Structure (New York: Gordon and Breach)

[100] Meakin C A and Arnett D 2007 Astrophys. J. 667448

[101] Viallet M, Meakin C, Arnett D and Mocák M 2013 The Astrophysical Journal 7691

[102] Herwig F, Woodward P R, Lin P H, Knox M and Fryer C 2014 Astrophys. J. Lett. 792 L3

[103] Woodward P R, Herwig F and Lin P H 2015 Astrophys. J. 79849

[104] Couch S M, Chatzopoulos E, Arnett W D and Timmes F X 2015 Astrophys. J. Lett. 808 L21

[105] Arnett W D and Meakin C 2016 Reports on Progress in Physics 79102901

[106] Chatzopoulos E, Couch S M, Arnett W D and Timmes F X 2016 Astrophys. J. 82261

[107] Müller B, Viallet M, Heger A and Janka H T 2016 Astrophys. J. 833124

[108] Cristini A, Meakin C, Hirschi R, Arnett D, Georgy C, Viallet M and Walkington I 2017 Mon. Not. Roy. Astron. Soc. $471279-300$

[109] Collins C, Müller B and Heger A 2017 ArXiv e-prints:1709.00236

[110] Goldreich P and Kumar P 1990 Astrophys. J. 363 694-704

[111] Kovalenko I G and Eremin M A 1998 Mon. Not. Roy. Astron. Soc. 298 861-870

[112] Foglizzo T and Tagger M 2000 Astron. and Astrophys. 363 174-183

[113] Foglizzo T 2001 Astron. and Astrophys. 368 311-324

[114] Lai D and Goldreich P 2000 Astrophys. J. 535402

[115] Cao Y and Lou Y Q 2010 Mon. Not. Roy. Astron. Soc. 403 491-495

[116] Takahashi K and Yamada S 2014 Astrophys. J. 794162

[117] Müller B, Melson T, Heger A and Janka H T 2017 Mon. Not. Roy. Astron. Soc. 472 491-513

[118] Kovasznay L S G 1953 Journal of the Aeronautical Sciences 20 657-674

[119] Woosley S E and Heger A 2007 Phys. Rep. 442269

[120] Abdikamalov E, Zhaksylykov A, Radice D and Berdibek S 2016 Mon. Not. Roy. Astron. Soc. $4613864-3876$

[121] Chatzopoulos E, Graziani C and Couch S M 2014 Astrophys. J. 79592

[122] Sagaut P and Cambon C 2008 Homogeneous Turbulence Dynamics (Cambridge University Press)

[123] Ryu J and Livescu D 2014 Journal of Fluid Mechanics 756 R1

[124] Mahesh K, Moin P and Lele S K 1996 The interaction of a shock wave with a turbulent shear flow Tech. Rep. TF-69 Thermosciences division, Department of Mechanical Engineering, Stanford University

[125] Wouchuk J G, Huete Ruiz de Lira C and Velikovich A L 2009 Phys. Rev. E 79(6) 066315

[126] Huete C, Wouchuk J G and Velikovich A L 2012 Phys. Rev. E 85(2) 026312 
[127] Huete C, Snchez A L and Williams F A 2013 Physics of Fluids 25076105

[128] Huete C, Abdikamalov E and Radice D 2017 Submitted to MNRAS, arXiv:1709.07363

[129] Fernández R and Thompson C 2009 Astrophys. J. 6971827

[130] Nagakura H, Yamamoto Y and Yamada S 2013 Astrophys. J. 765123

[131] Janka H T 2012 Ann. Rev. Nuc. Par. Sci. 62407

[132] Takahashi K, Iwakami W, Yamamoto Y and Yamada S 2016 Astrophys. J. 83175

[133] Epstein R I 1979 Mon. Not. Roy. Astron. Soc. 188305

[134] Burrows A 1987 Astrophys. J. Lett. 318 L57-L61

[135] Burrows A and Lattimer J M 1988 Phys. Rep. 163 51-62

[136] Burrows A and Fryxell B A 1993 Astrophys. J. Lett. 418 L33

[137] Nomoto K, Sugimoto D, Sparks W M, Fesen R A, Gull T R and Miyaji S 1982 Nature 299 803-805

[138] Nomoto K 1984 Astrophys. J. 277791

[139] Mezzacappa A, Calder A C, Bruenn S W, Blondin J M, Guidry M W, Strayer M R and Umar A S 1998 Astrophys. J. 493848

[140] Buras R, Janka H T, Rampp M and Kifonidis K 2006 Astron. and Astrophys. 457281

[141] Dessart L, Burrows A, Livne E and Ott C D 2006 Astrophys. J. 645534

[142] Roberts L F, Shen G, Cirigliano V, Pons J A, Reddy S and Woosley S E 2012 Phys. Rev. Lett. 108061103

[143] Wilson J R and Mayle R W 1988 Phys. Rep. 16363

[144] Wilson J R and Mayle R W 1993 Phys. Rep. 22797

[145] Schmitt R W 1995 Scientific American 272 70-75

[146] Bruenn S W and Dineva T 1996 Astrophys. J. Lett. 458 L71

[147] Bruenn S W, Raley E A and Mezzacappa A 2004 ArXiv e-prints:0404099

[148] Burrows A, Dessart L, Livne E, Ott C D and Murphy J 2007 Astrophys. J. 664 416-434

[149] Takiwaki T and Kotake K 2011 Astrophys. J. 74330

[150] Mösta P, Richers S, Ott C D, Haas R, Piro A L, Boydstun K, Abdikamalov E, Reisswig C and Schnetter E 2014 Astrophys. J. Lett. 785 L29

[151] Wheeler J C, Meier D L and Wilson J R 2002 Astrophys. J. 568807

[152] Akiyama S and Wheeler J C 2005 Astrophys. J. 629414

[153] Obergaulinger M, Cerdá-Durán P, Müller E and Aloy M A 2009 Astron. and Astrophys. 498 241

[154] Mösta P, Ott C D, Radice D, Roberts L F, Haas R and Schnetter E 2015 Nature 528376

[155] Endeve E, Cardall C Y, Budiardja R D and Mezzacappa A 2010 Astrophys. J. 7131219

[156] Obergaulinger M, Janka H T and Aloy M A 2014 Mon. Not. Roy. Astron. Soc. 4453169

[157] Cowling T G 1933 Monthly Notices of the Royal Astronomical Society 94 39-48

[158] Brandenburg A and Subramanian K 2005 Phys. Rep. 417 1-209

[159] Lopez L A and Fesen R A 2018 Space Science Reviews 21444

[160] Milisavljevic D and Fesen R A 2015 Science 347 526-530

[161] Fesen R A and Milisavljevic D 2016 Astrophys. J. 81817

[162] Fassia A and Meikle W P S 1999 Mon. Not. Roy. Astron. Soc. 302 314-320

[163] Hanuschik R W, Thimm G and Dachs J 1988 Mon. Not. Roy. Astron. Soc. 234 41P-49P

[164] Wang L, Wheeler J C, Höflich P, Khokhlov A, Baade D, Branch D, Challis P, Filippenko A V, Fransson C, Garnavich P, Kirshner R P, Lundqvist P, McCray R, Panagia N, Pun C S J, Phillips M M, Sonneborn G and Suntzeff N B 2002 Astrophys. J. 579 671-677

[165] Modjaz M, Kirshner R P, Blondin S, Challis P and Matheson T 2008 Astrophys. J. Lett. 687 L9 